\title{
Estimation of Timing Resolution for Very Fast Time-Of-Flight Detectors in Monte Carlo Simulations
}

\author{
Nikos Efthimiou, Member, IEEE, Elise Emond, Student Member, IEEE, Chris Cawthorne, \\ Charalampos Tsoumpas, Senior Member, IEEE and Kris Thielemans, Senior Member, IEEE
}

\begin{abstract}
In PET imaging the depth of absorption in the crystal contributes to the detection time uncertainty, which impacts the time resolution of the scatter. In addition, affects the nature of the timing distribution. It was found that when Photon Travel Spread (PTS) in the crystal is the only factor affecting the timing uncertainty, in which case, a Laplace kernel might describe the measured data, more accurately. It was shown that for crystals as thin as $20 \mathrm{~mm}$ the RMSE of the Laplace was smaller than that of a Normal. While when PTS is combined with an addition coincidence detection resolution (CDR) then, a Normal achieves better RMSE, but with dependency on the crystal size. Results in terms of CRC, of a simulated NEMA phantom, confirmed that reconstruction using a Laplace kernel can model the data better for thicker crystals.
\end{abstract}

Index Terms-GATE, STIR, Scintillation Crystals, Time-OfFlight, PET, LYSO

\section{INTRODUCTION}

$\mathbf{T}$ IME-OF-FLIGHT (TOF) benefits on PET imaging sparked a race towards faster timing resolutions [1][4]. Monte Carlo (MC) simulations are frequently used to investigate the benefits of TOF in image quality [5]-[7], investigate the performance of novel detectors and scintillation crystals [8]. The impact of TOF reconstruction with suboptimal or incorrect kernels has been discussed in the past [9]. It was shown that it impacts the Contrast Recovery (CR) is the most important consequence.

The GATE Monte Carlo (MC) simulation toolkit [10] allows the user to set the detector's timing resolution

Manuscript received February 15, 2019. This project was supported by the European Cooperation for Science and Technology Action TD1401: Fast Advanced Scintillation Timing (http://cern.ch/fast-cost), GlaxoSmithKline R\&D (BIDS3000030921), the Daisy Appeal Charity and the CCP PET-MR (EPSRC grant EP/M022587/1).We thank Dr. Assem Allam and his family for the generous donation to help found the PET Research Centre at the University of Hull and for their continued support. Elise Emond wishes to thank Yusheng $\mathrm{Li}$ for discussions on the model.

Nikos Efthimiou is with the PET Research Centre, Faculty of Health Sciences, University of Hull, HU6 7RX Hull, UK (e-mail: n.efthymiou@ @ull.ac.uk).

Elise Emond is with the Institute of Nuclear Medicine, University College London, London NW1 2BU, UK (e-mail: elise.emond.16@ucl.ac.uk).

Chris Cawthorne is with the School of Life Sciences, Faculty of Health Sciences, University of Hull, Hull HU6 7RX, UK (e-mail: c.cawthorne@ hull.ac.uk).

Charalampos Tsoumpas is with the Department of Biomedical Imaging Science, University of Leeds, Leeds LS2 9JT, UK (e-mail: c.tsoumpas@leeds.ac.uk).

Kris Thielemans is with the Institute of Nuclear Medicine, University College London, London NW1 2BU, UK (e-mail: k.thielemans@ucl.ac.uk).
(setTimeResolution) on each detector, individually. Effectively this macro-command configures the Coincidence Detection Resolution (CDR) of the system, as two detectors are in-coincidence. However, fitting a Gaussian kernel on the detection time difference between the two $\gamma$-photon, revealed significant differences in the simulated timing resolution $\left(\mathrm{FWHM}_{\mathrm{TSF}}\right)$.

As has been presented [11], at high timing resolutions the contribution of Photon Travel Spread (PTS) inside the crystal is not negligible. Crystals as thin as $3 \mathrm{~mm}$ could see an impact of $20 \mathrm{ps}$ in their CDR [2].

Although, studies on the timing limitations of the scintillating crystals [12] and MC simulations of the optical light transport, have been presented [8], to the best of our knowledge the effect of photon absorption depth on the TOF kernel and then on the reconstructed images has not been studied. In particular, the effects of the time modelling in the sensitive detector in GATE simulations has not been investigated in detail.

Recent modifications to STIR [13] which add TOF reconstruction [7] allow investigation of the image quality using different TOF kernels. The aforementioned extra kernel options will be published as open source code with the rest of the library.

\section{Materials AND Methods}

\section{A. MC scanner model}

The geometry of a cylindrical PET scanner was simulated using the GATE simulation toolkit (v.8.1) [14]. The scanner was comprised of 666 detectors per ring, on 24 rings. The inner ring radius was $424.5 \mathrm{~mm}$. The crystals were made by LYSO $\left(4 \times 4 \mathrm{~mm}^{2}\right)$, with density $7.105 \mathrm{~g} / \mathrm{cm}^{3}$. This configuration provided realistic characteristics, similar to the PreLude 420 by Saint Gobain [15]. Crystal thicknesses under test were 20 and $40 \mathrm{~mm}$. The energy resolution was set to $14 \%$ and the energy window was $435-650 \mathrm{keV}$. The coincidence time window was $4.1 \mathrm{~ns}$. Two CDR configurations were considered (a) ideal $\mathrm{CDR}=0 \mathrm{ps}$ and (b) non-ideal $\mathrm{CDR}=35.35 \mathrm{ps}$, described in detail in later paragraphs.

\section{B. $\gamma$-photon detection}

$511 \mathrm{keV} \gamma$-photons propagating through matter interact mainly in means of photoelectric effect and Compton scattering. The intensity of a narrow monochromatic $\gamma$-photon 
beam is described by the exponential model $I=I_{0} \exp (-\mu L)$, where $\mu\left(\mathrm{cm}^{-1}\right)$ is the linear attenuation coefficient.

The Depth Of Interaction (DOI) if expressed in terms of time is correlated to the PTS and introduces uncertainty in the detection time of the photon. In this case let $I=I_{0} \exp (-\lambda T)$, where $\lambda=2 \mu c$ in $\left(\mathrm{ps}^{-1}\right)$ with $c \approx 0.2998(\mathrm{~mm} / \mathrm{ps})$ the speed of light and $T=L / c$ (ps) the maximum time that a $\gamma$-photon can travel in the crystal before get absorbed or escape from the back.

In PET imaging the two $\gamma$-photons are in coincidence, therefore their joined probability is described by the Laplace distribution [?] $\left(f_{L}\right)$ and let $F_{L}$ be the corresponding Cumulative Distribution Function (CDF).

The effect of DOI in the crystal on the scanner's timing performance was investigated using a thin cylindrical rod, with radius $0.5 \mathrm{~mm}$, emitting back-to-back $511 \mathrm{keV} \gamma$-photons. The rod was thin in order to minimize the error of the origin of the two photons. The polar angles of the emitted photons were restricted to perpendicular to the $\mathrm{z}$ axis, in order to reduce the contribution of oblique incident photons. The simulation duration was $100 \mathrm{~s}$, adequate for good statistics under this setup.

Two TOF scanner configurations were considered, (1) The CDR of the detectors set to $0 \mathrm{ps}$ which would simulate a scanner with ideal timing response and (2) the non-ideal timing response on which the CDR of the scanner was set to $50 \mathrm{ps}$.

1) Ideal timing response: In this case, the distance the $\gamma$ photons travel inside the crystal is the only factor contributing to the detection timing uncertainty of the scanner. The PDF of the timing response, for different crystal lengths is shown in Fig. 1. Similar distributions have been presented previously [16].

Using Maximum Likelihood Estimate (MLE) a $f_{L}$ and a $f_{N}$ distributions were fitted on the simulated timing responses. The $\mathrm{FWHM}_{\mathrm{T}}$ of the $f_{N}$ was found 51.95 and 68.64 ps for the 20 and $40 \mathrm{~mm}$ crystal, respectively. This proves that the $\mathrm{FWHM}_{\mathrm{T}}$ depend on the size of the crystal.

The root mean square errors (RMSE) for $f_{N}$ was 0.0273 and 0.0421 , while for $f_{L}$ were 0.0178 and 0.0031 for 20 and $40 \mathrm{~mm}$, respectively. This showed that as the crystal size got larger the Laplace distribution described the data better.

For LYSO, the characteristic long tails of the Laplace distribution were suppressed at thickness of $20 \mathrm{~mm}$. For given a material the truncation point depends only on the crystal's length. As such, the Laplace distribution is able to describe the $40 \mathrm{~mm}$ events much better then the $20 \mathrm{~mm}$. In all cases the $f_{L}$ under-estimated the peaks and over-estimated the tails.

2) Non-Ideal timing response: In this case, the timing response of the scanner depended on the PTS and the time resolution of the detectors. As in paragraph II-B1 a $f_{N}$ and a $f_{L}$ were fitted on the data.

The $\mathrm{FWHM}_{\mathrm{T}}$ of the $f_{N}$ was found 73.85 and 86.7 ps for the 20 and $40 \mathrm{~mm}$ crystal, respectively.

The RMSE for $f_{N}$ was 0.0045 and 0.0093 , while for $f_{L}$ were 0.0325 and 0.0177 for 20 and $40 \mathrm{~mm}$, respectively. The $f_{N}$ demonstrated smaller error in this case. However, $f_{N}$

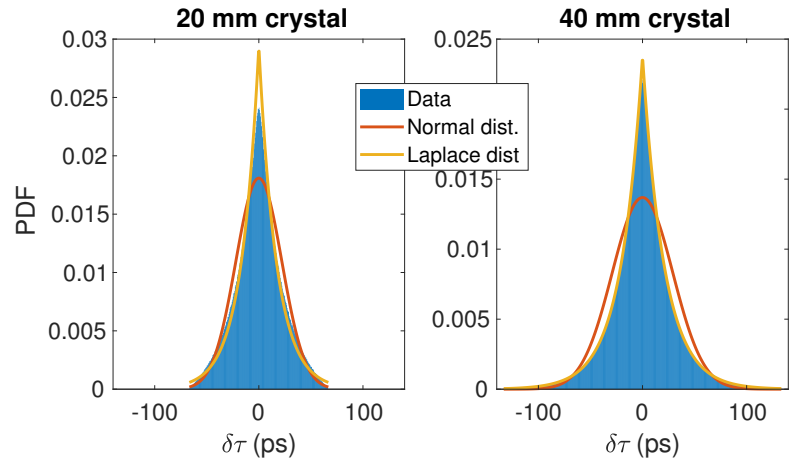

Fig. 1. Fitting of a Normal and a Laplace distributions on the timing responses for crystal lengths $20,40 \mathrm{~mm}$ with CDR set to $0.0 \mathrm{ps}$.

achieved the best RMSE with the thin crystal and the $f_{L}$ with the thick. Which indicated the underlying contributions of the two distribution, contributing to the total response kernel.

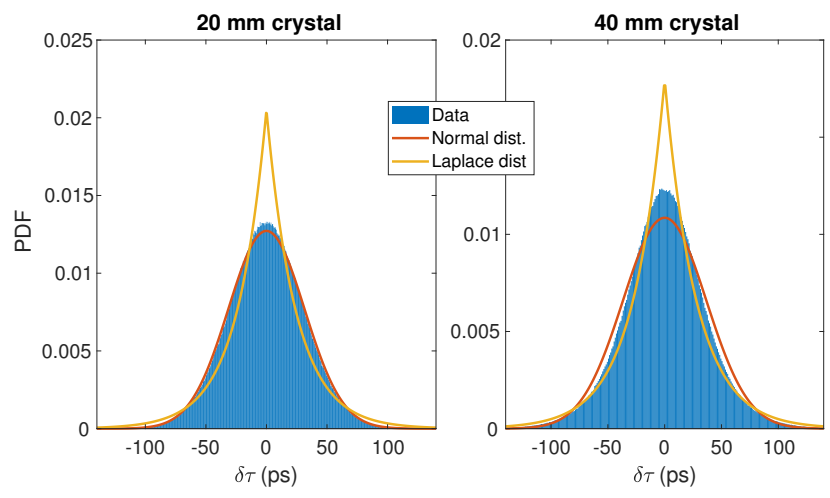

Fig. 2. Timing response for crystals with length (a) 20 and $40 \mathrm{~mm}$ with $C D R=50$ ps. A Laplace and a Normal distribution were fitted on the data, using MLE.

\section{NEMA phantom}

A NEMA Image Quality phantom [17] was simulated with GATE (v.8.1) and reconstructed with STIR, using TOFListmode MLEM. All datasets had 20E6 true events, as all phantom scattered (on the phantomSD volumes) and randoms were excluded from the reconstruction. The average DOI for each scanner template was modified to reflect the different crystal size. This was determined by examining the simulated data.

1) Figures of merit: The images were evaluated in terms of contrast recovery coefficient (CRC), calculated as:

$$
C R C_{r}=\frac{\left(\frac{\mu_{H, r}}{\mu_{B, r}}-1\right)}{\alpha-1}
$$

were $\alpha=4.19$ for sphere $r, \mu_{H, r}$ is the mean value in the ROI and $\mu_{B, r}$ the mean value in the background. The ROIs were selected according to the NEMA protocol [17]. 


\section{RESULTS}

1) Ideal timing response: As shown on Figure 3, for the largest hot sphere $(11 \mathrm{~mm})$, in the case of $20 \mathrm{~mm}$ crystal both the Gaussian and Laplace kernel overestimated CRC. This is an indication of model miss-match between the measured data and the kernel used in the reconstruction. In $40 \mathrm{~mm}$ crystal the Gaussian kernel overestimated the CRC, too, however the Laplace achieved $90 \%$ CRC. Moreover, after 75 iterations only the Laplacian kernel on $40 \mathrm{~mm}$ crystal managed to converge.

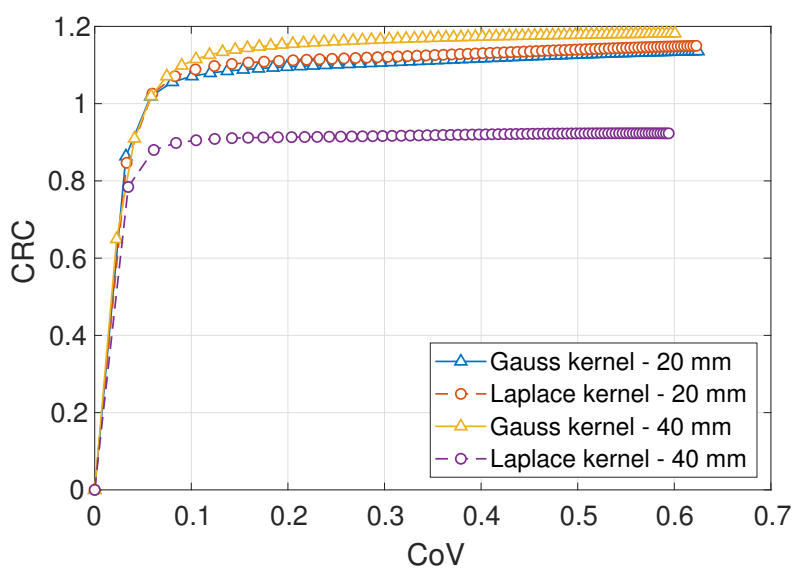

Fig. 3. Contrast recovery coefficient over coefficient of variation, for the largest hot sphere $(11 \mathrm{~mm})$, ideal timing response and combinations of TOF kernel type and crystal sizes.

In Figure 4 the corresponding reconstructed images, are displayed. The iteration number was chosen to be that of $99 \%$ convergence (wherever possible), has been achieved. As such, for reconstruction with the Gaussian kernel, the $40^{\text {th }}$ and $29^{\text {th }}$ for the $20 \mathrm{~mm}$ and $40 \mathrm{~mm}$ crystals, respectively. While for the Laplace the corresponding iteration number were the $39^{\text {th }}$ and $16^{\text {th }}$.

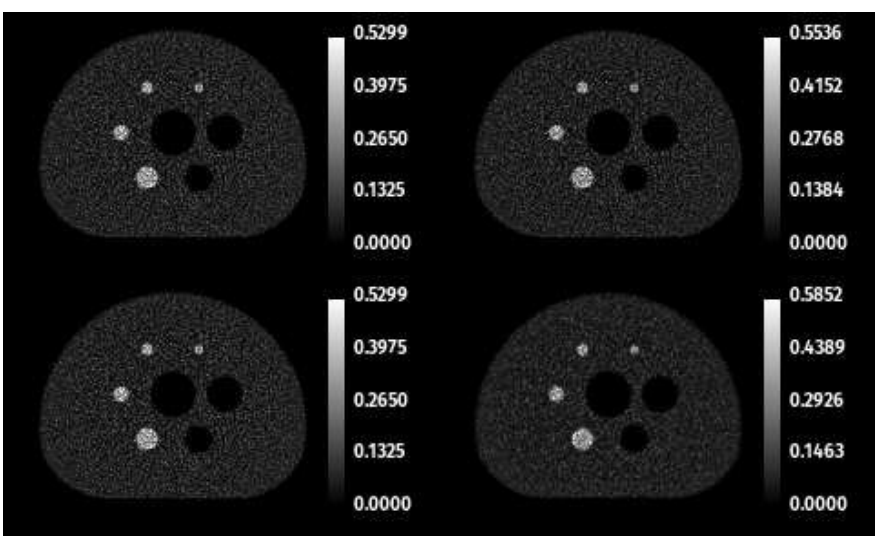

Fig. 4. Reconstructed images for all combinations using Gaussian kernel (top row) and Laplace kernel (bottom row) for $20 \mathrm{~mm}$ (first column) and $40 \mathrm{~mm}$ (second column).

2) Non-Ideal timing response: $\mathrm{CR}$ with $\mathrm{CDR}=50.0 \mathrm{ps}$ CRC of largest hot sphere $(11 \mathrm{~mm})$, is shown on Figure 5. The results show that in the case of the $20 \mathrm{~mm}$ crystal the Gaussian kernel, and for the $40 \mathrm{~mm}$ the Laplace kernel, provided $100 \%$ convergence. While the Laplace over-estimated the $20 \mathrm{~mm}$ crystal and the Gaussian did not converge for the $40 \mathrm{~mm}$.

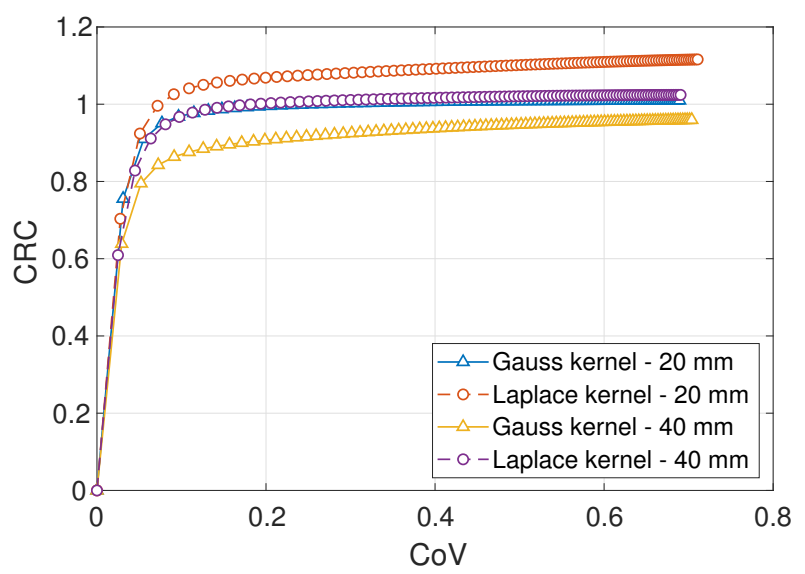

Fig. 5. Contrast recovery coefficient over iteration number for $\mathrm{FWHM}_{\mathrm{CDR}}=50 \mathrm{ps}$, crystal sizes 10,20 and $40 \mathrm{~mm}$, reconstructed with Normal-Normal $\left(f_{N N}\right)$ and Normal-Laplace $\left(f_{N L}^{*}\right)$ convolution kernels.

\section{DISCUSSION}

Including the effect of the photon travel spread in the crystal in the system's timing modelling in STIR reconstruction, was presented. In very high TOF resolutions the detection time uncertainty associated with the $\gamma$-photon's different absorption depths inside the crystal affects the distribution of the gammaphotons' distribution.

As the probability absorption in the crystal is an exponential distribution, the two in-coincidence crystals shape to a Laplacian distribution (double exponential).

When the crystal thickness is thin (accounting for the crystal's material properties), the tails of the Laplacian distribution are truncated. Then a Gaussian function might describe better the measurements [16]. The cause of a Laplace distribution for the modelling of the system's timing resolution is in very good agreement, with the simulations for crystals $20 \mathrm{~mm}$ onward.

In terms of CRC the interpretation of the results in not straight forward. In the ideal case, an over-estimation in contrast is demonstrated for the thinner crystal under both kernels. This is an indication that the reconstruction model does not describe the measurements properly. On the other hand, the $40 \mathrm{~mm}$ in combination with the Laplace kernel provides $90 \%$ CRC, which is very good. The appropriateness of the Laplace kernel for the thick crystal was anticipated, as the fitting demonstrated lower errors.

On the non-ideal case the Gaussian kernel performs better for the thinner crystal and the Laplace on the thicker. Note that that in the case of the thick crystal, the difference between the Gaussian and the Laplace kernel was small. However, a Laplace kernel should not be used with the thinner crystal, as over-estimation of the contrast, was presented.

Current investigation, has significant limitations which we will address in the future. Such as the size of the crystal usually is not long enough to absorb all incident photons, the exponential distribution in used is truncated and therefore 
the Laplace distribution is doubly-truncated. In addition, we believe the combined effect of the LTS and CDR would be best described by a convolution between the two models rather than either.

\section{CONCLUSiON}

The use of non-Gaussian timing kernel for TOF reconstruction, was investigate using Monte-Carlo simulations. The shape of the scanner's timing responses depends on the crystal properties and can be approximated a Laplacian distribution in very fast coincidence detection resolutions and thick crystals. The use of a Laplacian kernel under these settings might impact positively the image quality in terms of contrast recovery.

\section{REFERENCES}

[1] P. Lecoq, "Pushing the Limits in Time-of-Flight PET Imaging," IEEE Trans Rad Plasma Med Sci, vol. 1, no. 6, pp. 473-485, 112017.

[2] M. V. Nemallapudi, S. Gundacker, P. Lecoq, E. Auffray, A. Ferri, A. Gola, and C. Piemonte, "Sub-100 ps coincidence time resolution for positron emission tomography with LSO:Ce codoped with Ca," Phys. Med. Biol., vol. 60, no. 12, pp. 4635-4649, 62015.

[3] C. Dujardin, E. Auffray, E. Bourret-Courchesne, P. Dorenbos, P. Lecoq, M. Nikl, A. N. Vasil'ev, A. Yoshikawa, and R.-Y. Zhu, "Needs, Trends, and Advances in Inorganic Scintillators," IEEE Trans. Nucl. Sci., vol. 65, no. 8, pp. 1977-1997, 82018.

[4] B. F. Hutton, K. Erlandsson, and K. Thielemans, "Advances in clinical molecular imaging instrumentation," Clinical and Translational Imaging, vol. 6, no. 1, pp. 31-45, 2 2018. [Online]. Available: http://link.springer.com/10.1007/s40336-018-0264-0

[5] E. Clementel, S. Vandenberghe, J. Karp, and Surti Suleman, "Evaluation of Image Signal-to-Noise Ratio in Time-of-flight PET," IEEE Nucl. Sci. Symp. Conf. Rec., pp. 3917-3921, 2011.

[6] N. Efthimiou, E. Emond, C. Cawthorne, C. Tsoumpas, and K. Thielemans, "Reconstruction of Time-of-Flight Projection Data with the STIR reconstruction framework," in 2017 IEEE Nuclear Science Symposium and Medical Imaging Conference (NSS/MIC). IEEE, 10 2017, pp. 1-3.

[7] N. Efthimiou, E. Emond, P. Wadhwa, C. Cawthorne, C. Tsoumpas, and K. Thielemans, "Implementation and validation of time-of-flight PET image reconstruction module for listmode and sinogram projection data in the STIR library," Phys. Med. Biol., 12 2018. [Online]. Available: http://iopscience.iop.org/article/10.1088/1361-6560/aaf9b9

[8] E. Berg, E. Roncali, and S. R. Cherry, "Optimizing light transport in scintillation crystals for time-of-flight PET: an experimental and optical Monte Carlo simulation study." Biomed. Opt. Express, vol. 6, no. 6, pp. 2220-30, 62015.

[9] M. Daube-Witherspoon, S. Surti, S. Matej, M. Werner, S. Jayanthi, and J. S. Karp, "Influence of Time-of-Flight kernel accuracy in TOF-PET reconstruction," IEEE Nucl. Sci. Symp. Conf. Rec., vol. 3, pp. 17231727, 2007.

[10] S. Jan, G. Santin, D. Strul, S. Staelens, and K. Assi, "GATE : a simulation toolkit for PET and SPECT," Phys. Med. Biol., vol. 49, pp. 4543-4561, 2004.

[11] S. Gundacker, A. Knapitsch, E. Auffray, P. Jarron, T. Meyer, and P. Lecoq, "Time resolution deterioration with increasing crystal length in a TOF-PET system," Nucl. Instrum. Methods Phys. Res A, vol. 737, pp. $92-100,2014$.

[12] J. W. Cates, R. Vinke, and C. S. Levin, "Analytical calculation of the lower bound on timing resolution for PET scintillation detectors comprising high-aspect-ratio crystal elements." Physics in medicine and biology, vol. 60, no. 13, pp. 5141-61, 72015.

[13] K. Thielemans, C. Tsoumpas, S. Mustafovic, T. Beisel, P. Aguiar, N. Dikaios, and M. W. Jacobson, "STIR: Software for tomographic image reconstruction release 2," Phys. Med. Biol, vol. 57, no. 4, pp. 867-883, 22012.

[14] J. Strydhorst and I. Buvat, "Redesign of the GATE PET coincidence sorter," Physics in Medicine and Biology, vol. 61, no. 18, pp. N522N531, 92016

[15] S. Gobain, "Saint-Gobain Crystals - Innovative Materials and Applications solutions," Tech. Rep. [Online]. Available: https://www. crystals.saint-gobain.com/
[16] V. C. Spanoudaki and C. S. Levin, "Investigating the temporal resolution limits of scintillation detection from pixellated elements: comparison between experiment and simulation," Phys. Med. Biol., vol. 56, no. 3, pp. 735-756, 22011.

[17] A. M. Grant, T. W. Deller, M. M. Khalighi, S. H. Maramraju, G. Delso, and C. S. Levin, "NEMA NU 2-2012 performance studies for the SiPMbased ToF-PET component of the GE SIGNA PET/MR system," Med. Phys., vol. 43, no. 5, pp. 2334-2343, 42016. 